\title{
Discovery and Resolution of Anomalies in Web Access Control Policies
}

\author{
Hongxin Hu, Member, IEEE, Gail-Joon Ahn, Senior Member, IEEE, and Ketan Kulkarni
}

\begin{abstract}
Emerging computing technologies such as web services, service-oriented architecture, and cloud computing has enabled us to perform business services more efficiently and effectively. However, we still suffer from unintended security leakages by unauthorized actions in business services while providing more convenient services to Internet users through such a cutting-edge technological growth. Furthermore, designing and managing web access control policies are often error-prone due to the lack of effective analysis mechanisms and tools. In this paper, we represent an innovative policy anomaly analysis approach for web access control policies, focusing on extensible access control markup language policy. We introduce a policy-based segmentation technique to accurately identify policy anomalies and derive effective anomaly resolutions, along with an intuitive visualization representation of analysis results. We also discuss a proof-of-concept implementation of our method called XAnalyzer and demonstrate how our approach can efficiently discover and resolve policy anomalies.
\end{abstract}

Index Terms-Access control policies, XACML, conflict, redundancy, discovery and resolution

\section{INTRODUCTION}

W ITH the tremendous growth of web applications and web services deployed on the Internet, the use of a policy-based approach has recently received considerable attention to accommodate the security requirements covering large, open, distributed, and heterogeneous computing environments. eXtensible Access Control Markup Language (XACML) [25], which is a general-purpose access control policy language standardized by the Organization for the Advancement of Structured Information Standards, has been broadly adopted to specify access control policies for various applications, especially web services [28]. In an XACML policy, multiple rules may overlap, which means one access request may match several rules. Moreover, multiple rules within one policy may conflict, implying that those rules not only overlap each other, but also yield different decisions. Conflicts in an XACML policy may lead to both safety problem (e.g., allowing unauthorized access) and availability problem (e.g., denying legitimate access).

An intuitive means for resolving policy conflicts by a policy designer is to remove all conflicts by modifying the policies. However, resolving conflicts through changing the policies is notably difficult, even impossible, in practice from many aspects. First, the number of conflicts in an XACML policy is potentially large, since an XACML policy may consist of hundreds or thousands of rules.

- H. Hu is with the Department of Computer and Information Sciences, Delaware State University, 1200 N. DuPont Highway, Dover, DE 19901. E-mail: hhu@desu.edu.

- G.-J. Ahn is with the Security Engineering for Future Computing Laboratory, and the Ira A. Fulton School of Engineering, Arizona State University, PO Box 878809, Tempe, AZ 85287. E-mail: gahn@asu.edu.

- K. Kulkarni is with NVIDIA, Sunnyvale, CA 94086. E-mail: ketankulkarni29@gmail.com.

Manuscript received 27 Mar. 2012; revised 9 Oct. 2012; accepted 23 Jan. 2013; published online 14 Mar. 2013.

For information on obtaining reprints of this article, please send e-mail to: tdsc@computer.org, and reference IEEECS Log Number TDSC-2012-03-0062. Digital Object Identifier no. 10.1109/TDSC.2013.18.
Second, conflicts in XACML policies are probably very complicated, because one rule may conflict with multiple other rules, and one conflict may be associated with several rules. Besides, an XACML policy for a distributed application may be aggregated from multiple parties. Also, an XACML policy may be maintained by more than one administrator. Without a priori knowledge on the original intentions of policy specification, changing a policy may affect the policy's semantics and may not resolve conflicts correctly. Furthermore, in some cases, a policy designer may intentionally introduce certain overlaps in XACML policy components by implicitly reflecting that only the first rule is important. In this case, conflicts are not an error, but intended, which would not be necessary to be changed.

Since the conflicts in XACML policies always exist and are hard to be eliminated, XACML defines four different combining algorithms to automatically resolve conflicts [25]: Deny-Overrides, Permit-Overrides, First-Applicable, and Only-One-Applicable. Unfortunately, XACML currently lacks a systematic mechanism for precisely detecting conflicts. Identifying conflicts in XACML policies is critical for policy designers since the correctness of selecting a combining algorithm for an XACML policy or policy set component heavily relies on the information from conflict diagnosis. Without precise conflict information, the effectiveness of combining algorithms for resolving policy conflicts cannot be guaranteed.

Another critical problem for XACML policy analysis is redundancy discovery and removal. A rule in an XACML policy is redundant if every access request that matches the rule also matches other rules with the same effect. As the response time of an access request largely depends on the number of rules to be parsed within a policy, redundancies in a policy may adversely affect the performance of policy evaluation. Therefore, policy redundancy is treated as policy anomaly as well. Redundancy elimination 
can be regarded as one of effective solutions for optimizing XACML policies and improving the performance of XACML evaluation.

Policy anomaly detection has recently received a great deal of attention [3], [13], [23], [29], especially, in firewall policy analysis. Corresponding policy analysis tools, such as Firewall Policy Advisor [3], FIREMAN [29], and FAME [13], with the goal of discovering firewall policy anomalies have been developed. However, we cannot directly adopt those approaches for analyzing XACML policy anomalies due to several reasons. First, most previous approaches are mainly capable of detecting pairwise policy anomalies. However, it is necessary for a complete anomaly detection to consider all policy components as a whole piece. In other words, previous policy anomaly analysis approaches are still needed to be improved [4]. Second, the structure of firewall policies is flat, while XACML has a hierarchical structure supporting recursive policy specification. Third, a firewall policy only supports one conflict resolution strategy (first-match) to resolve conflicts but XACML has four rule/policy combining algorithms. Additionally, a firewall rule is typically specified with fixed fields, while an XACML rule can be multivalued. Therefore, a new policy analysis mechanism is desirable to fulfill the requirements from anomaly analysis aspects in XACML policies.

In this paper, we introduce a policy-based segmentation technique, which adopts a binary decision diagram (BDD)based data structure to perform set operations, for policy anomaly discovery and resolution. Based on this technique, an authorization space defined by an XACML policy or policy set component can be divided into a set of disjoint segments. Each segment associated with a unique set of XACML components indicates an overlapping relation (either conflicting or redundant) among those components. Accurate anomaly information is crucial to the success of anomaly resolution. For example, conflict diagnosis information provided by a policy analysis tool can be utilized to guide the policy designers in selecting appropriate combining algorithms. Moreover, we present a grid-based representation technique to show policy anomaly diagnosis information in an intuitive manner, facilitating more efficient policy anomaly management. Besides, we observe that current XACML conflict resolution mechanisms are too restrictive by applying only one combining algorithm to resolve all identified conflicts within an XACML policy or policy set component. Also, many other desirable conflict resolution strategies exist [14], [16], [17], but cannot be directly supported by XACML. Thus, we additionally provide a flexible and extensible policy conflict resolution method in this paper. In addition, based on our policybased segmentation technique, we provide an effective redundancy discovery mechanism, where both rule redundancies within one policy and redundancies across multiple policies or policy sets can be detected and eliminated. Furthermore, we implement a policy analysis tool XAnalyzer based on our approach along with extensive experiments.

The rest of this paper is organized as follows: Section 2 briefly discusses anomalies in XACML policies. We describe the underlying data structure for XACML representation based on BDDs in Section 3. Section 4 presents our conflict detection and resolution approaches. In Section 5, we address our redundancy discovery and removal approaches. In Section 6, we discuss the implementation of our tool XAnalyzer and the evaluation of our approach. Section 7 overviews the related work, and we conclude this paper in Section 8.

\section{BACKGROUND}

\subsection{Overview of XACML}

XACML has become the de facto standard for describing access control policies and offers a large set of built-in functions, data types, combining algorithms, and standard profiles for defining application-specific features. At the root of all XACML policies is a policy or a policy set. A policy set is composed of a sequence of policies or other policy sets along with a policy combining algorithm and a target. A policy represents a single access control policy expressed through a target, a set of rules and a rule combining algorithm. The target defines a set of subjects, resources, and actions the policy or policy set applies to. A rule set is a sequence of rules. Each rule consists of a target, a condition, and an effect. The target of a rule determines whether an access request is applicable to the rule and it has a similar structure as the target of a policy or a policy set.

An XACML policy often has conflicting rules or policies, which are resolved by four different combining algorithms: Deny-Overrides, Permit-Overrides, First-Applicable, and OnlyOne-Applicable [25]. Fig. 1 shows an example XACML policy. The root policy set $P S_{1}$ contains two policies, $P_{1}$ and $P_{2}$, which are combined using First-Applicable combining algorithm. The policy $P_{1}$ has three rules, $r_{1}, r_{2}$, and $r_{3}$, and its rule combining algorithm is Deny-Overrides. The policy $P_{2}$ includes two rules $r_{4}$ and $r_{5}$ with Deny-Overrides combining algorithm. In this example, there are four subjects: Manager, Designer, Developer, and Tester; two resources: Reports and Codes; and two actions: Read and Change. Note that both $r_{2}$ and $r_{3}$ define conditions over the Time attribute.

\subsection{Anomalies in XACML Policies}

An XACML policy may contain both policy components and policy set components. Often, a rule anomaly occurs in a policy component, which consists of a sequence of rules. On the other hand, a policy set component consists of a set of policies or other policy sets; thus, anomalies may also arise among policies or policy sets. We address XACML policy anomalies at both policy level and policy set level:

- Anomalies at policy level. A rule is conflicting with other rules, if this rule overlaps with others but defines a different effect. For example, the deny rule $r_{1}$ is in conflict with the permit rule $r_{2}$ in Fig. 1 because rule $r_{2}$ allows the access requests from a designer to change codes in the time interval [8:00, 17:00], which are supposed to be denied by $r_{1}$; and a rule is redundant if there is other same or more general rules available that have the same effect. For instance, if we change the effect of $r_{2}$ to Deny, $r_{3}$ 


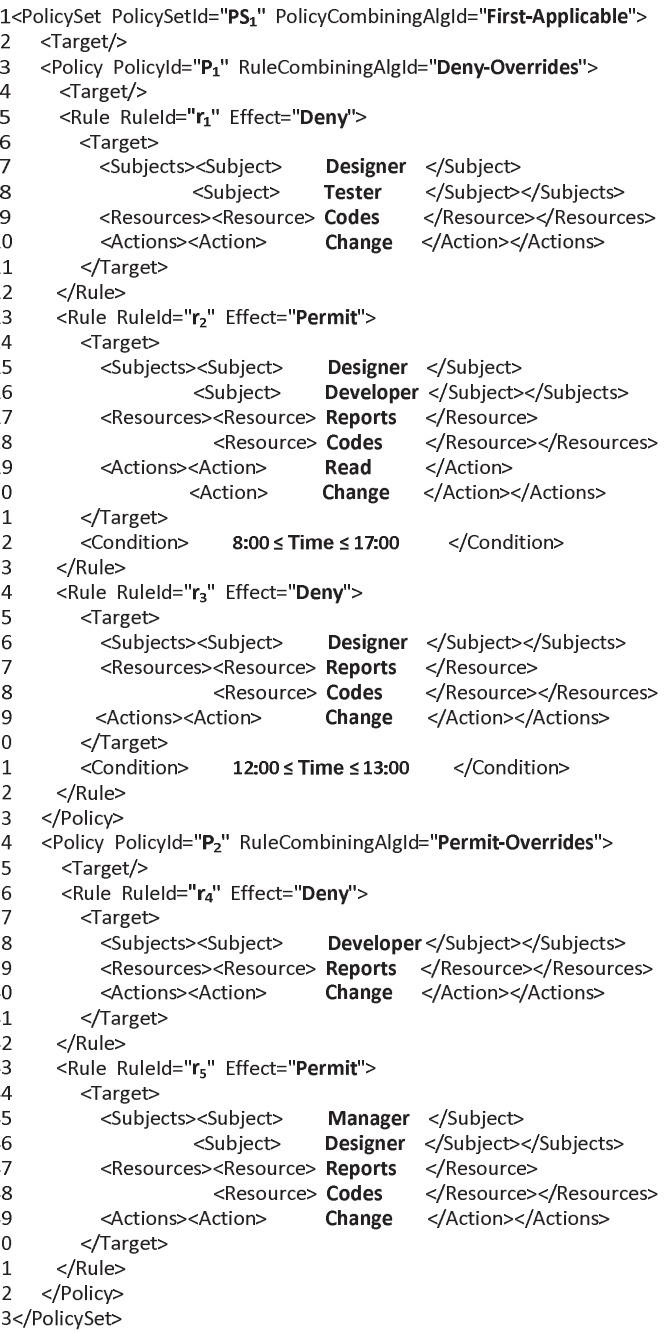

Fig. 1. An example XACML policy.

becomes redundant because $r_{2}$ will also deny a designer to change reports or codes in the time interval [12:00, 13:00].

- Anomalies at policy set level. Anomalies may also occur across policies or policy sets in an XACML policy. For example, considering two policy components $P_{1}$ and $P_{2}$ of the policy set $P S_{1}$ in Fig. 1, $P_{1}$ is conflicting with $P_{2}$, because $P_{1}$ permits the access requests that a developer changes reports in the time interval [8:00, 17:00], which are denied by $P_{2}$. On the other hand, $P_{1}$ denies the requests allowing a designer to change reports or codes in the time interval [12:00, 13:00], which are permitted by $P_{2}$. Supposing the effect of $r_{2}$ is changed to Deny and the condition of $r_{2}$ is removed, $r_{4}$ is turned to be redundant with respect to $r_{2}$, even though $r_{2}$ and $r_{4}$ are placed in different policies $P_{1}$ and $P_{2}$, respectively.

A policy anomaly may involve in multiple rules. For example, in Fig. 1, access requests that a designer changes codes in the time interval [12:00,13:00] are permitted by $r_{2}$, but denied by both $r_{1}$ and $r_{3}$. Thus, this conflict associates with three rules. For another example, suppose the effect of $r_{3}$ is changed to Permit and the subject of $r_{3}$ is replaced by
Manager and Developer. If we only examine pairwise redundancies, $r_{3}$ is not a redundant rule. However, if we check multiple rules simultaneously, we can identify $r_{3}$ is redundant considering $r_{2}$ and $r_{5}$ together. We observe that precise anomaly diagnosis information is crucial for achieving an effective anomaly resolution. In this paper, we attempt to design a systematic approach and corresponding tool not only for accurate anomaly detection but also for effective anomaly resolution.

\section{Underlying Data Structure}

Our policy-based segmentation technique introduced in subsequent sections requires a well-formed representation of policies for performing a variety of set operations. BDD [9] is a data structure that has been widely used for formal verification and simplification of digital circuits. In this work, we leverage BDD as the underlying data structure to represent XACML policies and facilitate effective policy analysis.

Given an XACML policy, it can be parsed to identify subject, action, resource, and condition attributes. Once these attributes are identified, all XACML rules can be transformed into Boolean expressions [5]. Each Boolean expression of a rule is composed of atomic Boolean expressions combined by logical operators $\vee$ and $\wedge$. Atomic Boolean expressions are treated as equality constraints or range constraints on attributes (e.g., Subject $=$ "Designer") or on conditions (e.g., 8:00 $\leq$ Time $\leq 17: 00$ ).

Example 1. Consider the example XACML policy in Fig. 1 in terms of atomic Boolean expressions. The Boolean expression for rule $r_{1}$ is

$$
\begin{aligned}
\text { Subject } & =\text { "Designer" } \vee \text { Subject }=\text { "Tester" }) \\
& \wedge(\text { Resource }=" \text { Codes" }) \wedge(\text { Action }=" \text { Change" }) .
\end{aligned}
$$

The Boolean expression for rule $r_{2}$ is

$$
\begin{aligned}
& (\text { Subject }=" \text { Designer" } \vee \text { Subject }=" \text { Developer } ") \\
& \wedge(\text { Resource }=" \text { Reports" } \vee \text { Resource }=" \text { Codes" }) \\
& \wedge(\text { Action }=\text { "Read" } \vee \text { Action }=" \text { "Change" }) \\
& \wedge(8: 00 \leq \text { Time } \leq 17: 00)
\end{aligned}
$$

Boolean expressions for XACML rules may consist of atomic Boolean expressions with overlapping value ranges. In such cases, those atomic Boolean expressions are needed to be transformed into a sequence of new atomic Boolean expressions with disjoint value ranges. Agrawal et al. [1] have identified different categories of such atomic Boolean expressions and addressed corresponding solutions for those issues. We adopt similar approach to construct our Boolean expressions for XACML rules.

We encode each of the atomic Boolean expression as a Boolean variable. For example, an atomic Boolean expression Subject $=$ "Designer" is encoded into a Boolean variable $S_{1}$. A complete list of Boolean encoding for the example XACML policy in Fig. 1 is shown in Table 1. We then utilize the Boolean encoding to construct Boolean expressions in terms of Boolean variables for XACML rules. 
TABLE 1

Atomic Boolean Expressions and Corresponding Boolean Variables for $P_{1}$

\begin{tabular}{|l|c|}
\hline Unique Atomic Boolean Expression & Boolean Variable \\
\hline Subject $=$ "Designer" & $S_{1}$ \\
\hline Subject $=$ "Tester" & $S_{2}$ \\
\hline Subject $=$ "Developer" & $S_{3}$ \\
\hline Subject $=$ "Manager" & $S_{4}$ \\
\hline Resource = "Reports" & $R_{1}$ \\
\hline Resource = "Codes" & $R_{2}$ \\
\hline Action $=$ "Read" & $A_{1}$ \\
\hline Action $=$ "Change" & $A_{2}$ \\
\hline 8:00 $\leq$ Time $<12: 00$ & $C_{1}$ \\
\hline $12: 00 \leq$ Time $<13: 00$ & $C_{2}$ \\
\hline $13: 00 \leq$ Time $\leq 17: 00$ & $C_{3}$ \\
\hline
\end{tabular}

Example 2. Consider the example XACML policy in Fig. 1 in terms of Boolean variables. The Boolean expression for rule $r_{1}$ is

$$
\left(S_{1} \vee S_{2}\right) \wedge\left(R_{2}\right) \wedge\left(A_{2}\right)
$$

The Boolean expression for rule $r_{2}$ is

$$
\left(S_{1} \vee S_{3}\right) \wedge\left(R_{1} \vee R_{2}\right) \wedge\left(A_{1} \vee A_{2}\right) \wedge\left(C_{1} \vee C_{2} \vee C_{3}\right) \text {. }
$$

BDDs are acyclic directed graphs that represent Boolean expressions compactly. Each nonterminal node in a BDD represents a Boolean variable and has two edges with binary labels, 0 and 1 for nonexistent and existent, respectively. Terminal nodes represent Boolean value $\mathrm{T}$ (True) or $\mathrm{F}$ (False). Figs. $2 \mathrm{a}$ and $2 \mathrm{~b}$ give $\mathrm{BDD}$ representations of two rules $r_{1}$ and $r_{2}$, respectively.

Once the BDDs are constructed for XACML rules, performing set operations, such as unions $(\cup)$, intersections $(\cap)$, and set differences $(\backslash)$, required by our policybased segmentation algorithms (see Algorithms 1 and 2) is efficient as well as straightforward. Fig. 2c shows an integrated BDD, which is the difference of $r_{2}{ }^{\prime}$ BDD from $r_{1}{ }^{\prime}$ BDD $\left(r_{2} \backslash r_{1}\right)$. Note that the resulting BDDs from the set operations may have less number of nodes due to the canonical representation of BDD.

\section{Conflict Detection and Resolution}

We first introduce a concept of authorization space, which adopts the aforementioned BDD-based policy representation to perform policy anomaly analysis. This concept is defined as follows:

Definition 1 (Authorization space). Let $R_{x}, P_{x}$, and $P S_{x}$ be the set of rules, policies, and policy sets, respectively, of an XACML policy $x$. An authorization space for an XACML policy component $c \in R_{x} \cup P_{x} \cup P S_{x}$ represents a collection of all access requests ${ }^{1} Q_{c}$ to which a policy component $c$ is applicable.

\subsection{Conflict Detection Approach}

Our conflict detection mechanism examines conflicts at both policy level and policy set level for XACML policies. To precisely identify policy conflicts and facilitate an effective conflict resolution, we present a policy-based segmentation

1. We only consider single-valued requests in this definition. Multivalued requests are discussed in Section 5 .
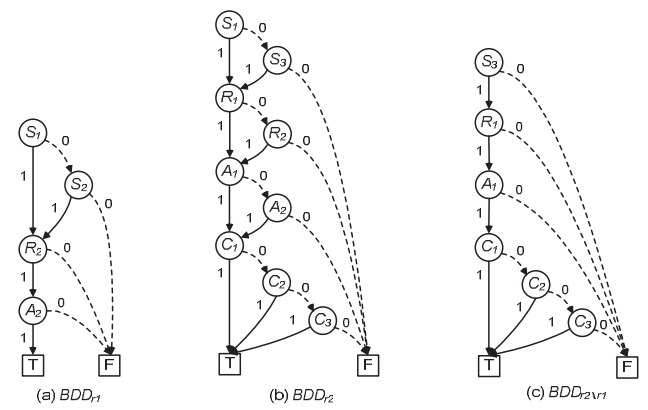

Fig. 2. Representing and operating on rules of XACML policy with BDD.

technique to partition the entire authorization space of a policy into disjoint authorization space segments. Then, conflicting authorization space segments (called conflicting segment in the rest of this paper), which contain policy components with different effects, are identified. Each conflicting segment indicates a policy conflict.

\subsubsection{Conflict Detection at Policy Level}

A policy component in an XACML policy includes a set of rules. Each rule defines an authorization space with the effect of either permit or deny. We call an authorization space with the effect of permit permitted space and an authorization space with the effect of deny denied space.

Algorithm 1 shows the pseudocode of generating conflicting segments for a policy component $P$. An entire authorization space derived from a policy component is first partitioned into a set of disjoint segments. As shown in lines 17-33 in Algorithm 1, a function called Partition () accomplishes this procedure. This function works by adding an authorization space $s$ derived from a rule $r$ to an authorization space set $S$. A pair of authorization spaces must satisfy one of the following relations: subset (line 19), superset (line 24), partial match (line 27), or disjoint (line 32). Therefore, one can utilize set operations to separate the overlapped spaces into disjoint spaces.

Conflicting segments are identified as shown in lines 6-10 in Algorithm 1. A set of conflicting segments $C S$ : $\left\{c s_{1}, c s_{2}, \ldots, c s_{n}\right\}$ from conflicting rules has the following three properties:

1. All conflicting segments are pairwise disjoint: $c s_{i} \cap c s_{j}=\emptyset, 1 \leq i \neq j \leq n$;

2. any two different requests $q$ and $q^{\prime}$ within a single conflicting segment $\left(c s_{i}\right)$ are matched by exact same set of rules: $\operatorname{Get} \operatorname{Rule}(q)=\operatorname{Get} \operatorname{Rule}\left(q^{\prime}\right),{ }^{2} \forall q \in c s_{i}, q^{\prime} \in$ $c s_{i}, q \neq q^{\prime} ;$ and

3. the effects of matched rules in any conflicting segments contain both "Permit" and "Deny."

To facilitate the correct interpretation of analysis results, a concise and intuitive representation method is necessary. For the purposes of brevity and understandability, we first employ a two-dimensional geometric representation for each authorization space segment. Note that a rule in an XACML policy typically has multiple fields; thus, a complete representation of authorization space should be multidimensional. Also, we utilize colored rectangles to

2. GetRule() is a function that returns all rules matching a request. 


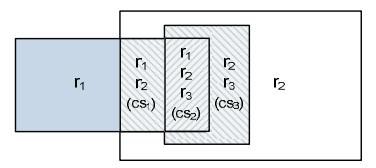

$\square$ Permitted Space $\square$ Denied Space $\square$ Conflicting Space (a) Disjoint segments of authorization space for policy $P_{1}$

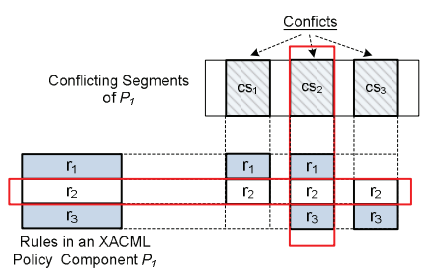

(b) Grid representation of policy conflict in policy $P_{1}$
Fig. 3. Authorization space representation for policy $P_{1}$ in the example XACML policy.

denote two kinds of authorization spaces: permitted space (white color) and denied space (gray color), respectively. Fig. 3a gives a representation of the segments of authorization space derived from the policy $P_{1}$ in the XACML example policy shown in Fig. 1. We can notice that five unique disjoint segments are generated. In particular, three conflicting segments $c s_{1}, c s_{2}$, and $c s_{3}$ are identified, representing three policy conflicts.

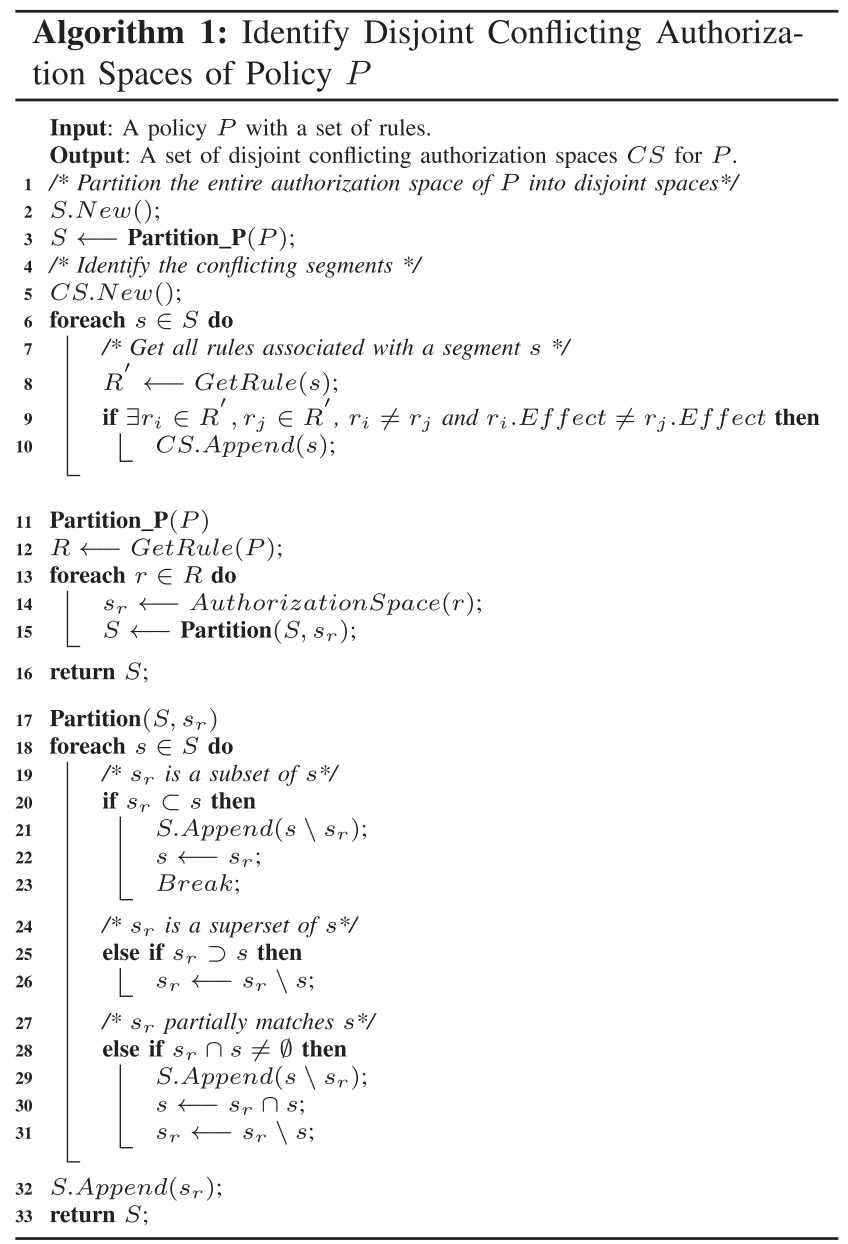

When a set of XACML rules interacts, one overlapping relation may be associated with several rules. Meanwhile, one rule may overlap with multiple other rules and can be involved in a couple of overlapping relations (overlapping segments). Different kinds of segments and associated rules can be viewed like in Fig. 3a. However, it is still difficult for a policy designer or administrator to figure out how many segments one rule is involved in. To address the need of a more precise conflict representation, we additionally introduce a grid representation that is a matrix-based visualization of policy conflicts, in which space segments are displayed along the horizontal axis of the matrix, rules are shown along the vertical axis, and the intersection of a segment and a rule is a grid that displays a rule's subspace covered by the segment.

Fig. $3 \mathrm{~b}$ shows a grid representation of conflicts in the policy $P_{1}$ in our example policy. We can easily determine which rules are covered by a segment, and which segments are associated with a rule. For example, as shown in Fig. 3b, we can notice that a conflicting segment $c s_{2}$, which points out a conflict, is related to a rule set consisting of three rules $r_{1}, r_{2}$, and $r_{3}$ (highlighted with a horizontal red rectangle), and a rule $r_{2}$ is involved in three conflicting segments $c s_{1}$, $c s_{2}$, and $c s_{3}$ (highlighted with a vertical red rectangle). Our grid representation provides a better understanding of policy conflicts to policy designers and administrators with an overall view of related segments and rules.

\subsubsection{Conflict Detection at Policy Set Level}

There are two major challenges that need to be taken into consideration when we design an approach for XACML analysis at policy set level:

1. XACML supports four rule/policy combining algorithms: First-Applicable, Only-One-Applicable, DenyOverrides, and Permit-Overrides.

2. An XACML policy is specified recursively and, therefore, has a hierarchical structure. In XACML, a policy set contains a sequence of policies or policy sets, which may further contain other policies or policy sets.

Each authorization space segment also has an effect, which is determined by the XACML components covered by this segment. For nonconflicting segments, the effect of a segment equals to the effect of components covered by this segment. Regarding conflicting segments, the effect of a segment depends on the following four cases of combining algorithm $(\mathcal{C} \mathcal{A})$, which is used by the owner (a policy or a policy set) of the segment:

1. $\mathcal{C A}=$ First-Applicable. In this case, the effect of a conflicting segment equals to the effect of the first component covered by the conflicting segment.

2. $\mathcal{C A}=$ Permit-Overrides. The effect of a conflicting segment is always assigned with "Permit," since there is at least one component with "Permit" effect within this conflicting segment.

3. $\mathcal{C A}=$ Deny-Overrides. The effect of a conflicting segment always equals to "Deny."

4. $\mathcal{C} \mathcal{A}=$ Only-One-Applicable. The effect of a conflicting segment equals to the effect of only applicable component.

To support the recursive specifications of XACML policies, we parse and model an XACML policy as a tree structure [20], [21]. Algorithm 2 shows the pseudocode of identifying disjoint conflicting authorization spaces for a policy set $P S$. To partition authorization spaces of all nodes contained in a policy set tree, this algorithm recursively calls the partition functions, Partition_P() and Partition_PS(), to deal with the policy nodes (lines 16-17) and the policy set nodes (lines 19-20), 


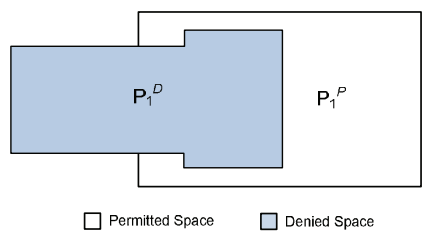

Fig. 4. Aggregation of authorization spaces for policy $P_{1}$ in the example XACML policy.

respectively. Once all children nodes of a policy set are partitioned, we can then represent the authorization space of each child node $(E)$ with two subspaces permitted subspace $\left(E^{P}\right)$ and denied subspace $\left(E^{D}\right)$ by aggregating all "Permit" segments and "Deny" segments, respectively, as follows:

$$
\begin{cases}E^{P}=\bigcup_{s_{i} \in S_{E}} s_{i} & \text { if } \operatorname{Effect}\left(s_{i}\right)=\text { Permit } \\ E^{D}=\bigcup_{s_{i} \in S_{E}} s_{i} & \text { if } \operatorname{Effect}\left(s_{i}\right)=\operatorname{Deny}\end{cases}
$$

where $S_{E}$ denotes the set of authorization space segments of the child node $E$.

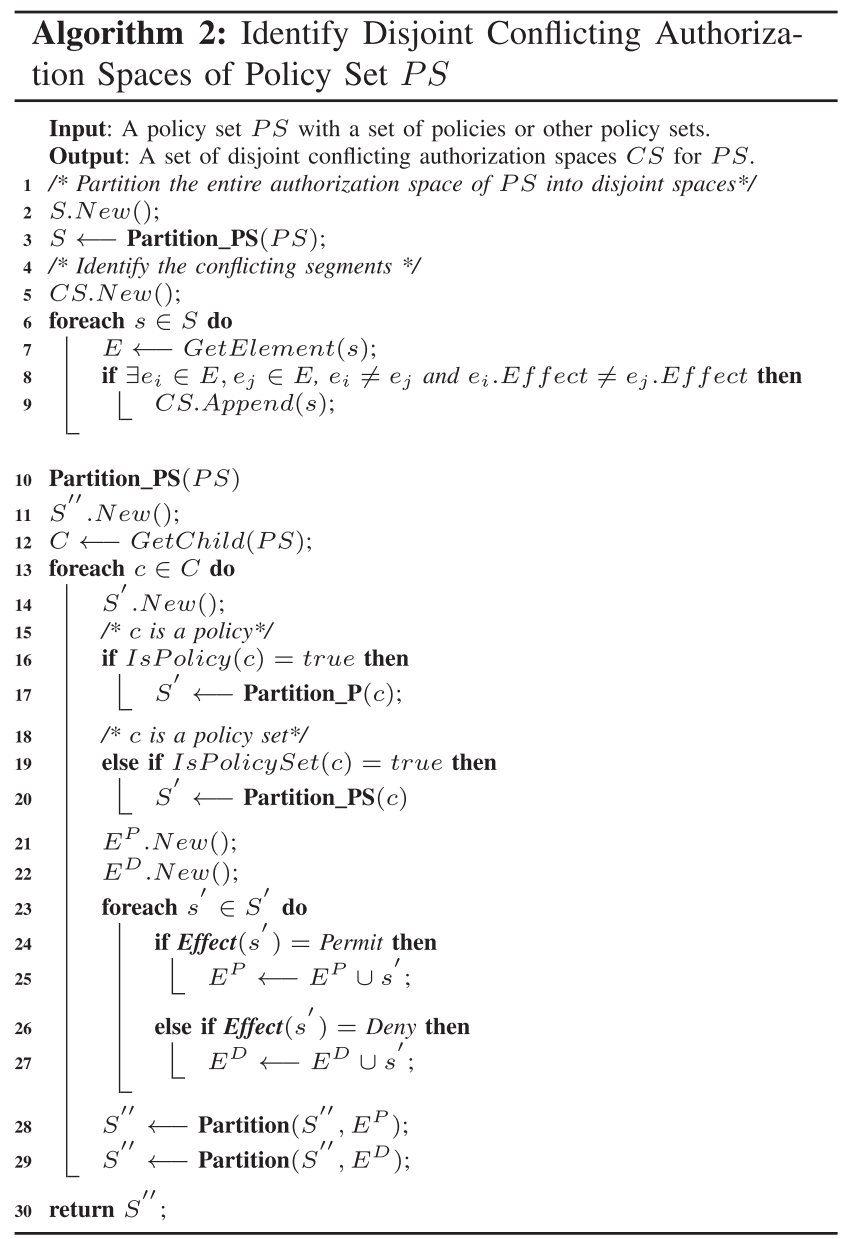

For example, since the combining algorithm of the policy $P_{1}$ in our example XACML policy is Deny-Overrides, the effects of three conflicting segments shown in Fig. 3 are "Deny." Fig. 4 shows the result of aggregating authorization spaces of the policy $P_{1}$, where two subspaces $P_{1}^{P}$ and $P_{1}^{D}$ are constructed.

To generate segments for the policy set $P S$, we can then leverage two subspaces $\left(E^{P}\right.$ and $\left.E^{D}\right)$ of each child node $(E)$

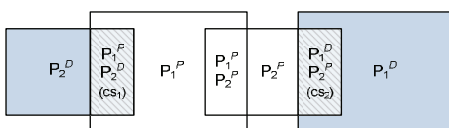

$\square$ Permitted Space $\square$ Denied Space $\square$ Confflicting Space

(a) Disjoint segments of authorization space for policy set $P S_{1}$

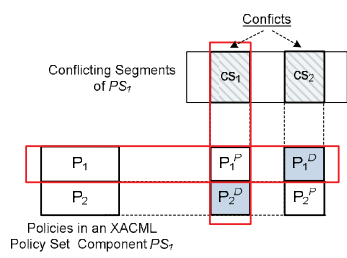

(b) Grid representation of policy conflicts in policy set $P S_{1}$
Fig. 5. Authorization space representation for policy set $P S_{1}$ in the example XACML policy.

to partition existing authorization space set belonging to $P S$ (lines 28-29). Fig. 5a represents an example of the segments of authorization space derived from policy set $P S_{1}$ in our example policy (Fig. 1). We can observe that seven unique disjoint segments are generated, and two of them $c s_{1}$ and $c s_{2}$ are conflicting segments. We additionally give a grid representation of conflicts in the policy set $P S_{1}$ shown in Fig. $5 \mathrm{~b}$. Then, we can easily identify that the conflicting segment $c s_{1}$ is related to two subspaces: $P_{1}$ 's permitted subspace $P_{1}^{P}$ and $P_{2}$ 's denied subspace $P_{2}^{D}$, and the policy $P_{1}$ is associated with two conflicts, where $P_{1}$ 's permitted subspace $P_{1}^{P}$ is involved in the conflict represented by $c s_{1}$ and $P_{1}$ 's denied subspace $P_{1}^{D}$ is related to the conflict represented by $c s_{2}$.

\subsection{Fine-Grained Conflict Resolution}

Once conflicts within a policy component or policy set component are identified, a policy designer can choose appropriate conflict resolution strategies to resolve those identified conflicts. However, current XACML conflict resolution mechanisms have limitations in resolving conflicts effectively. First, existing conflict resolution mechanisms in XACML are too restrictive and only allow a policy designer to select one combining algorithm to resolve all identified conflicts within a policy or policy set component. A policy designer may want to adopt different combining algorithms to resolve different conflicts. Second, XACML offers four conflict resolution strategies. However, many conflict resolution strategies exist [14], [17] but cannot be specified in XACML. Thus, it is necessary to seek a comprehensive conflict resolution mechanism for more effective conflict resolution. Toward this end, we introduce a flexible and extensible conflict resolution framework to achieve a fine-grained conflict resolution as shown in Fig. 6.

\subsubsection{Effect Constraint Generation from a Conflict Resolution Strategy}

Our conflict resolution framework introduces an effect constraint that is assigned to each conflicting segment. An effect constraint for a conflicting segment defines a desired response (either permit or deny) that an XACML policy should take when any access request matches the conflicting segment. The effect constraint is derived from the conflict resolution strategy applied to the conflicting segment, using a similar process of determining the effect of a conflicting segment described in Section 4.1.2. A policy designer chooses an appropriate conflict resolution strategy for each identified conflict by examining the features of conflicting segment and associated conflicting components. In our conflict resolution framework, a policy designer is 


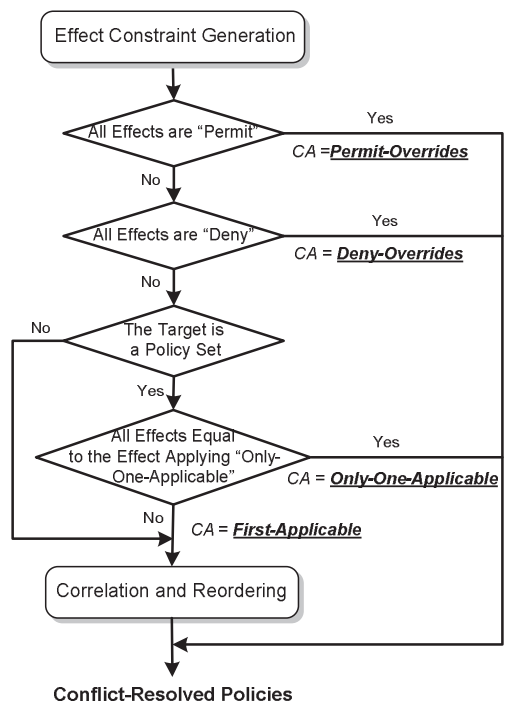

Fig. 6. Fine-grained conflict resolution framework.

able to adopt different strategies to resolve conflicts indicated by different conflicting segments. In addition to four standard XACML conflict resolution strategies, userdefined strategies [17], such as Recency-Overrides, SpecificityOverrides, and High-Majority-Overrides, can be implied in our framework as well. For example, applying a conflict resolution strategy, High-Majority-Overrides, to the second conflicting segment $c s_{2}$ of policy $P_{1}$ depicted in Fig. 3, an effect constraint Effect = "Deny" will be generated for $c s_{2}$.

\subsubsection{Conflict Resolution Based on Effect Constraints}

A key feature of adopting effect constraints in our framework is that other conflict resolution strategies assigned to resolve different conflicts by a policy designer can be automatically mapped to standard XACML combining algorithms, without changing the way that current XACML implementations perform. As illustrated in Fig. 6, an XACML combining algorithm can be derived for a target component by examining all effect constraints of the conflicting segments. If all effect constraints are "Permit," Permit-Overrides is selected for the target component to resolve all conflicts. In case all effect constraints are "Deny," Deny-Overrides is assigned to the target component. Then, if the target component is a policy set and all effect constraints can be satisfied by applying Only-OneApplicable combining algorithm, Only-One-Applicable is selected as the combining algorithm of the target component. Otherwise, First-Applicable is selected as the combining algorithm of the target component. To resolve all conflicts within the target component by applying FirstApplicable, the process of reordering conflicting components is compulsory to enable that the first-applicable component in each conflicting segment has the same effect with corresponding effect constraint.

Practically, one XACML component may get involved in multiple conflicts. In this case, removing such a component to satisfy one effect constraint may violate other effect constraints. Therefore, we cannot resolve a conflict individually by reordering a set of conflicting components associated with one conflict. On the other hand, it is also inefficient to deal with all conflicts together by reordering all conflicting components simultaneously. Thus, we next

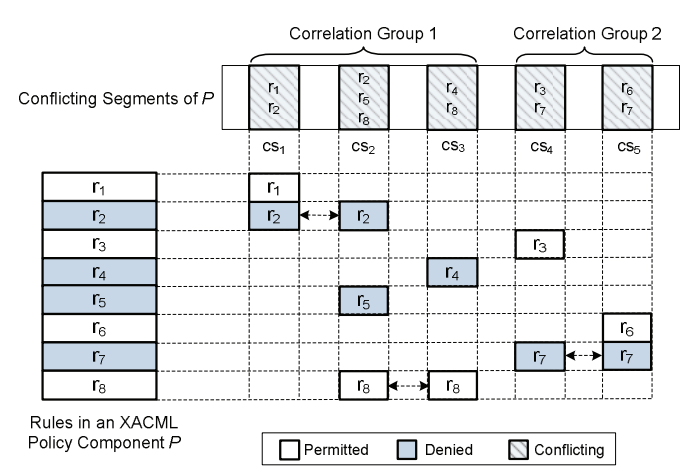

Fig. 7. Example of conflicting segment correlation.

introduce a correlation mechanism to identify dependent relationships among conflicting segments. The major benefit of identifying dependent relationships for a conflict resolution is to lessen the searching space of reordering conflicting components.

Fig. 7 shows an example for conflicting segment correlation, considering an XACML policy component $P$ with eight rules. Five conflicting segments are identified in this example. Several rules in this XACML policy component are involved in multiple conflicts. For example, $r_{2}$ contributes to two policy conflicts corresponding to two conflicting segments $c s_{1}$ and $c s_{2}$, respectively. Also, $r_{8}$ is associated with two conflicting segments $c s_{2}$ and $c s_{3}$. Suppose we want to satisfy the effect constraint of $c s_{2}$ by reordering associated conflicting rules, $r_{2}, r_{5}$, and $r_{8}$. The position change of $r_{2}$ and $r_{8}$ would affect conflicting segments, $c s_{1}$ and $c s_{2}$, respectively. Thus, a dependent relationship can be derived among $c s_{1}, c s_{2}$, and $c s_{3}$ with respect to the conflict resolution. Similarly, we can identify the dependent relation between $c s_{4}$ and $c s_{5}$. We organize those conflicting segments with a dependent relationship as a group called conflict correlation group. The pseudocode of an algorithm for identify conflict correlation groups is given in Algorithm 3.

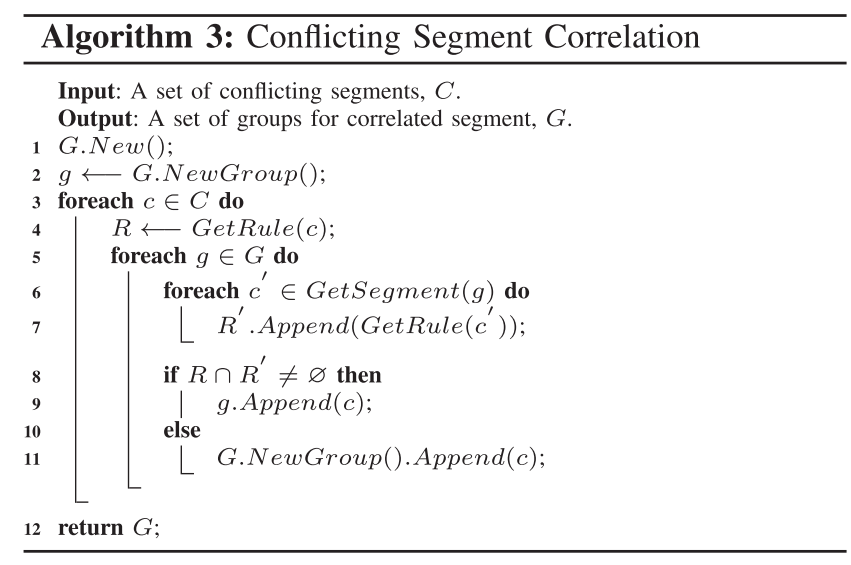

\section{Redundancy Discovery and Removal}

Our redundancy discovery and removal mechanism also leverage the policy-based segmentation technique to explore redundancies at both policy level and policy set level.

\subsection{Redundancy Elimination at Policy Level}

We employ following steps to identify and eliminate redundant rules at policy level. 


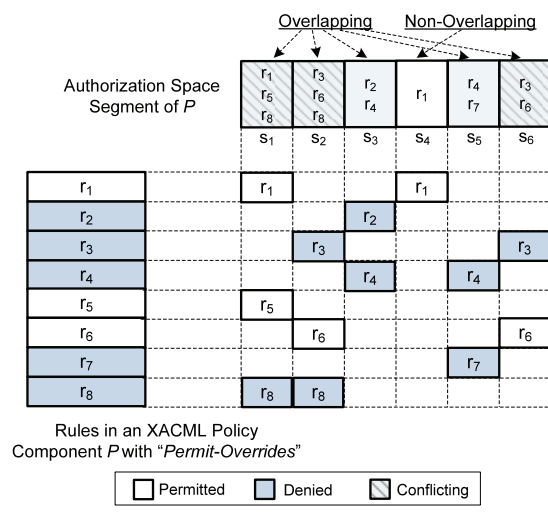

(a) Authorization space segmentation

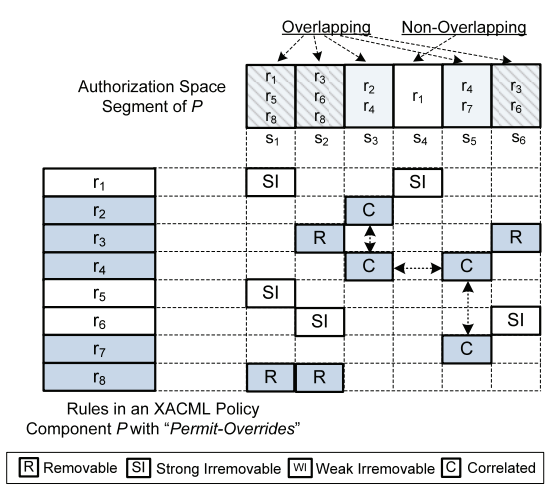

(b) Property assignment

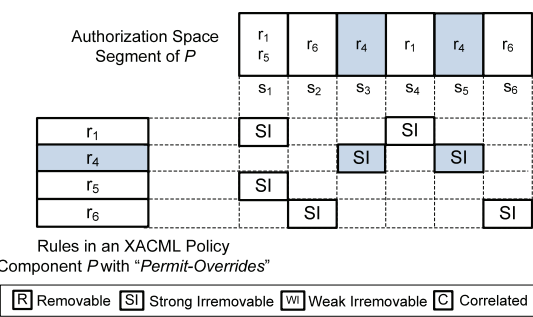

(c) Redundancy removal

Fig. 8. Example of eliminating redundancies at policy level.

\subsubsection{Authorization Space Segmentation}

We first perform the policy segmentation function Partition_P() defined in Algorithm 1 to divide the entire authorization space of a policy into disjoint segments. We classify the policy segments in following categories: nonoverlapping segment and overlapping segment, which is further divided into conflicting overlapping segment and nonconflicting overlapping segment. Each nonoverlapping segment associates with one unique rule, and each overlapping segment is related to a set of rules, which may conflict with each other (conflicting overlapping segment) or have the same effect (nonconflicting overlapping segment). Fig. 8a illustrates a grid representation of authorization space segmentation for a policy with eight rules. In this example, one policy segment $s_{4}$ is a nonoverlapping segment. Other policy segments are overlapping segments, including three conflicting overlapping segments $s_{1}, s_{2}$, and $s_{6}$, and two nonconflicting overlapping segments $s_{3}$ and $s_{5}$.

\subsubsection{Irremovable Rule Identification Considering Multivalued Requests}

An XACML request may be multivalued. For example, an XACML request can be "a person, who is both a Developer and a Designer, wants to change reports," where the subject has two values, Developer and Designer. A multivalued request may match several rules, which do not overlap with each other in terms of single-valued requests. For instance, the above multivalued request matches both $r_{4}$ and $r_{5}$ in the example policy shown in Fig. 1, although $r_{4}$ and $r_{5}$ have no overlapping relation considering single-valued requests. We observe that an XACML rule may be removable with respect to single-valued requests but irremovable taking into account multivalued requests. Therefore, we introduce a process to examine whether an XACML rule is irremovable considering multivalued requests based on the three cases of rule combining algorithm $(\mathcal{C A})$ :

1. $\mathcal{C} \mathcal{A}=$ First-Applicable. In this case, since a multivalued request may match the examined rule and any subsequent rule(s) in the policy, if there is a subsequent rule with a different effect, the examined rule is considered irremovable.
2. $\mathcal{C} \mathcal{A}=$ Permit-Overrides. A multivalued request may match the examined rule and any other rule(s) in the policy. If the examined rule is a "permit" rule and there is any other rule being a "deny" rule, the examined rule is irremovable.

3. $\mathcal{C} \mathcal{A}=$ Deny-Overrides. If the examined rule is a "deny" rule and there is any other rule being a "permit" rule, the examined rule is irremovable.

Algorithm 4 shows the pseudocode for the definition of a function Irremovablecheck ( ), which will subsequently be used in both property assignment and redundancy removal processes to check if a rule is irremovable in a policy considering multivalued requests.

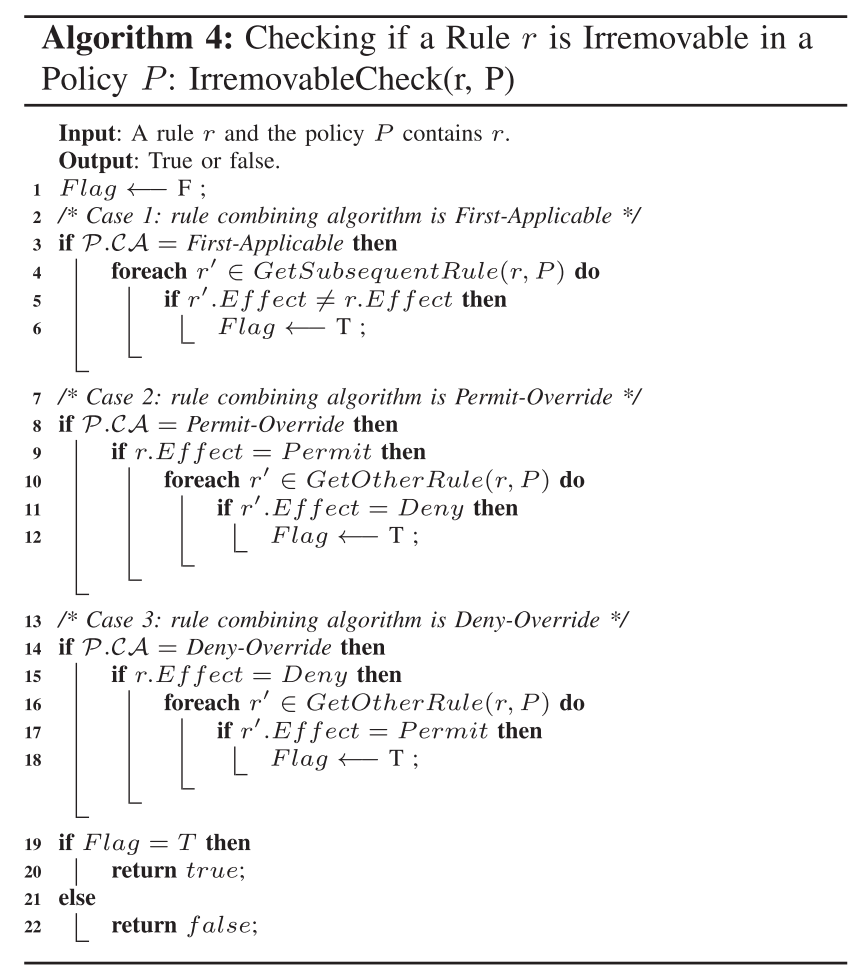

\subsubsection{Property Assignment for Rule Subspaces}

In this step, every rule subspace covered by a policy segment is assigned with a property. Four property values, removable $(\mathrm{R})$, strong irremovable (SI), weak irremovable (WI) and correlated (C), are defined to reflect different characteristics 
of rule subspace. $R$ property is used to indicate that a rule subspace is removable. In other words, removing such a rule subspace does not make any impact on the original authorization space of an associated policy. SI property means that a rule subspace cannot be removed, because 1) this rule subspace belongs to an irremovable rule with respect to multivalued requests, or 2) the effect of corresponding policy segment can be only decided by this rule. WI property is assigned to a rule subspace when any subspace belonging to the same rule has SI property. That means a rule subspace becomes irremovable due to the reason that other portions of this rule cannot be removed. $C$ property is assigned to multiple rule subspaces covered by a policy segment, if the effect of this policy segment can be determined by any of these rules. We next introduce four processes to perform the property assignments to all of rule subspaces within the segments of a policy, considering irremovable rules and different categories of policy segments.

Process 1: Property assignment for the rule subspace belonging to an irremovable rule. An irremovable rule can be identified by calling function Irremovablecheck (). All subspaces belonging to an irremovable rule are assigned with SI property.

Process 2: Property assignment for the rule subspace covered by a nonoverlapping segment. A nonoverlapping segment contains only one rule subspace. Thus, this rule subspace is assigned with SI property. Other rule subspaces associated with the same rule are assigned with WI property, excepting the rule subspaces that already have SI property.

Process 3: Property assignment for rule subspaces covered by a conflicting segment. We present this property assignment process based on the following three cases of rule combining algorithm:

1. $\mathcal{C} \mathcal{A}=$ First-Applicable. In this case, the first rule subspace covered by the conflicting segment is assigned with SI property. Other rule subspaces in the same segment are assigned with $R$ property. Meanwhile, other rule subspaces associated with the same rule are assigned with WI property except the rule subspaces already having SI property.

2. $\mathcal{C} \mathcal{A}=$ Permit-Overrides. All subspaces of "deny" rules in this conflicting segment are assigned with $R$ property. If there is only one "permit" rule subspace, this case is handled, which is similar to the First-Applicable case. If any "permit" rule subspace has been assigned with irremovable property, other rule subspaces without irremovable property are assigned with $R$ property. Otherwise, all "permit" rule subspaces are assigned with $C$ property.

3. $\mathcal{C} \mathcal{A}=$ Deny-Overrides. This case is dealt with as the same as Permit-Overrides case.

Process 4: Property assignment for rule subspaces covered by a nonconflicting overlapping segment. If any rule subspace has been assigned with irremovable property, other rule subspaces without irremovable property are assigned with $R$ property. Otherwise, all subspaces within the segment are assigned with $C$ property.

Fig. $8 \mathrm{~b}$ shows the result of applying our property assignment mechanism to the example presented in Fig. 8a. We can easily identify that $r_{3}$ and $r_{8}$ are removable

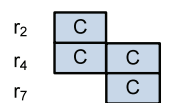

(a) Rule correlation

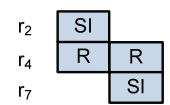

(b) Sequence one for correlation break

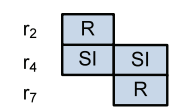

(c) Sequence two for correlation break
Fig. 9. Example of rule correlation break.

rules, where all subspaces are with $R$ property. However, we need to further examine the correlated rules $r_{2}, r_{4}$, and $r_{7}$, which contain subspaces with $C$ property.

\subsubsection{Rule Correlation Break and Redundancy Removal}

Rules covered by an overlapping segment are correlated with each other when the effect of the overlapping segment can be determined by any of those rules. Thus, keeping one correlated rule and removing others do not change the effect of the overlapping segment. In addition, some rules may get involved in multiple correlated relations. For example, in Fig. 8b, $r_{4}$ has two subspaces that are involved in the correlated relations with $r_{2}$ and $r_{7}$, respectively. Therefore, similar to the construction of conflict correlation groups, we build rule correlation groups based on these two situations so that dependent relationships among multiple correlated rules within one group can be examined simultaneously. For example, a correlation group consisting of three rules $r_{2}, r_{4}$, and $r_{7}$ can be identified in Fig. 8b.

The goal of rule correlation break is to discover as many redundant rules as possible. Different sequences to break rule correlations may lead to different results for redundancy removal. For example, Fig. 9a shows correlated relations of rules $r_{2}, r_{4}$, and $r_{7}$, and we can break their correlated relations into different sequences. As shown in Fig. 9b, if we first choose $r_{2}$ as an irremovable rule and assign $r_{2}{ }^{\prime}$ subspace SI property, only $r_{4}$ becomes an removable rule and $r_{7}$ is turned to be irremovable. However, as shown in Fig. 9c, if we first choose $r_{4}$ as an irremovable rule and assign two subspaces of $r_{4}$ with SI property, both $r_{2}$ and $r_{7}$ then become removable rules. To seek an optimal solution for rule correlation break, we measure a breaking degree for each correlated rule $r$, denoted as $B D(r)$, which indicates the number of removable rules if choosing $r$ as an irremovable rule. $B D(r)$ can be calculated with the following equation:

$$
B D(r)=\sum_{s_{i} \in C S(r)}\left(N C\left(s_{i}\right)-1\right),
$$

where function $C S(r)$ returns the set of all overlapping segments covering correlated subspaces of the rule $r$, and function $N C\left(s_{i}\right)$ returns the number of correlated rules covered by the segment $s_{i}$. For example, $C S\left(r_{4}\right)$ returns a segment set $\left\{s_{3}, s_{5}\right\}$ and $N C\left(s_{3}\right)$ equals to 2 . Since choosing $r$ as an irremovable rule turns the subspaces of other rules covered by the segment $s_{i}$ to removable, $N C\left(s_{i}\right)-1$ donates the number of these removable rules. Consequently, $B D(r)$ aggregates the number of removable rules if setting $r$ as irremovable. To maximize the number of removable rules for redundancy elimination, our correlation break process selects the rule with the maximum $B D$ value as the candidate irremovable rule each time. For instance, applying this equation to compute breaking degrees of three rules demonstrated in Fig. 9a, both $B D\left(r_{2}\right)$ and $B D\left(r_{7}\right)$ are equal to 1 , and $B D\left(r_{4}\right)$ is equal to 2 . Thus, we 
choose $r_{4}$ as the candidate irremovable rule in the first step for rule correlation break. Finally, two rules $r_{2}$ and $r_{7}$ become removable after breaking all correlations.

The pseudocode of the algorithm for eliminating redundancy at policy level is shown in Algorithm 5. Note that a function Irremovablecheck ( ) is called to check if a candidate rule is truly removable considering multivalued requests before removing the rule from the policy. Fig. $8 \mathrm{c}$ depicts the result of applying this algorithm to the example given in Fig. 8a. Four rules $r_{2}, r_{3}, r_{7}$, and $r_{8}$ were identified as redundant rules and removed from the policy. However, if we leverage the traditional redundancy detection method [23], [3], which was limited to detect pairwise redundancies, to this example, only two redundant rules $r_{2}$ and $r_{7}$ can be discovered.

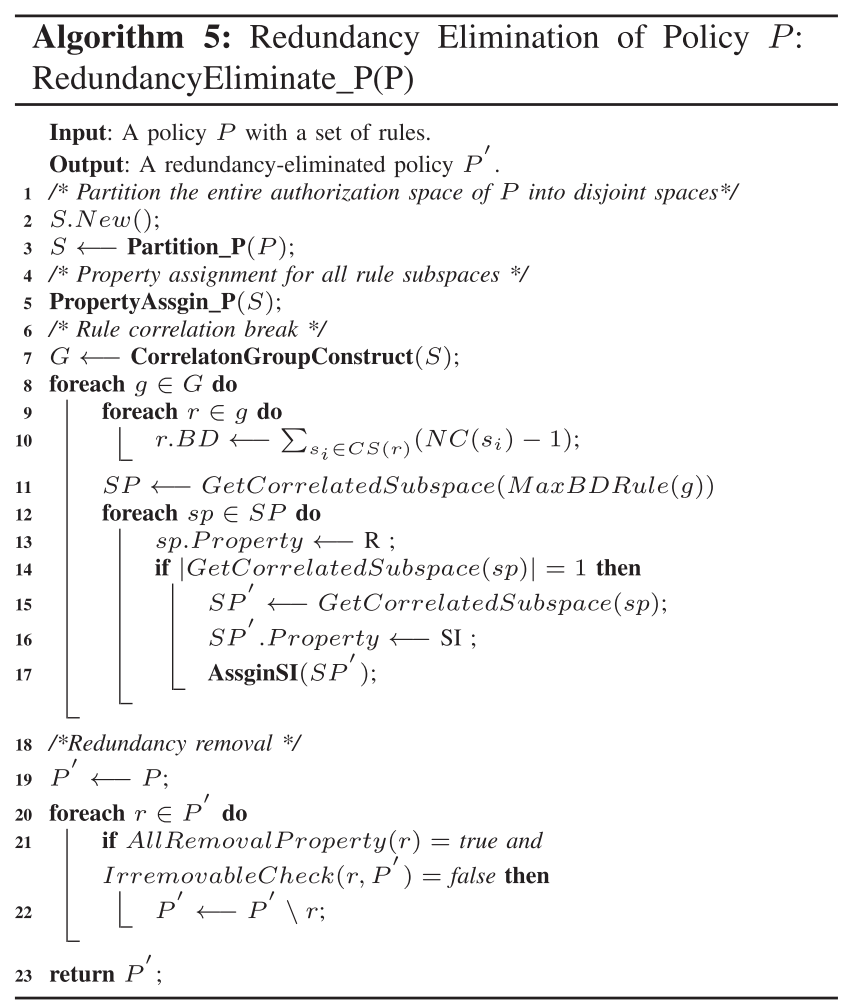

\subsection{Redundancy Elimination at Policy Set Level}

Similar to the solution of conflict detection at policy set level, we handle the redundancy removal for a policy set based on an XACML tree structure representation. If the children nodes of the policy set is a policy node in the tree, we perform RedundancyEliminate_P() function to eliminate redundancies. Otherwise, RedundancyEliminate_PS () function is excused recursively to eliminate redundancy in a policy set component.

After each component of a policy set $P S$ performs redundancy removal, the authorization space of $P S$ can be then partitioned into disjoint segments by performing Partition() function. Note that, in the solution for conflict detection at policy set level, we aggregate authorization subspaces of each child node before performing space partition, because we only need to identify conflicts among children nodes to guide the selection of policy combining algorithms for the policy set. However, for redundancy removal at policy set level, both redundancies

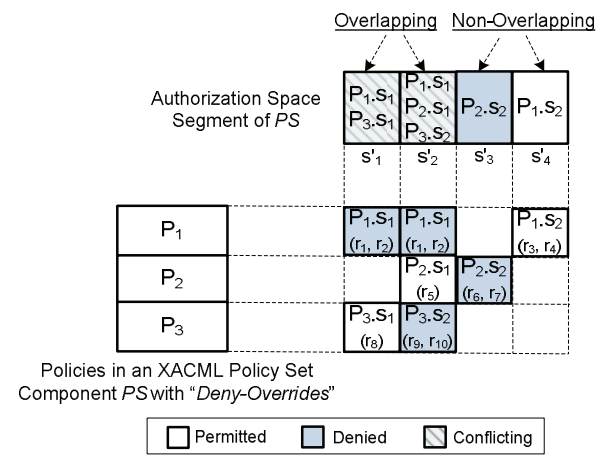

Fig. 10. Example of authorization space segmentation at policy set level for redundancy discovery and removal.

among children nodes and rule (leaf node) redundancies, which may exist across multiple policies or policy sets, should be discovered. Therefore, we keep the original segments of each child node and leverage those segments to generate the authorization space segments of PS. Fig. 10 demonstrates an example of authorization space segmentation of a policy set $P S$ with three children components $P_{1}$, $P_{2}$, and $P_{3}$. The authorization space segments of $P S$ are constructed based on the original segments of each child component. For instance, a segment $s_{2}^{\prime}$ of $P S$ covers three policy segments $P_{1} . s_{1}, P_{2} . s_{1}$, and $P_{3} . s_{2}$, where $P_{i} . s_{j}$ denotes that a segment $s_{j}$ belongs to a policy $P_{i}$.

The property assignment step at policy set level is similar to the property assignment step at policy level, except that the policy combining algorithm Only-One-Applicable needs to be taken into consideration at policy set level. The OnlyOne-Applicable case is handled similar to the First-Applicable case. We first check whether the combining algorithm is applicable. If the combining algorithm is applicable, the only applicable subspace is assigned with SI property. Otherwise, all subspaces within the policy set's segment are assigned with $R$ property.

After assigning properties to all segments of children components of $P S$, we next examine whether any child component is redundant. If a child component is redundant, this child component and all rules contained in the child component are removed from $P S$. Then, we examine whether there exist any redundant rules. In this process, the properties of all rule subspaces covered by a removable segment of a child component of $P S$ needs to be changed to removable. Note that when we change the property of a strong irremovable rule subspace to removable, other subspaces in the same rule with dependent WI property need to be changed to removable correspondingly.

\section{IMPLEMENTATION AND Evaluation}

We have implemented a policy analysis tool called XAnalyzer in Java. Based on our policy anomaly analysis mechanism, it consists of four core components: segmentation module, effect constraint generation module, strategy mapping module, and property assignment module. The segmentation module takes XACML policies as an input and identifies the authorization space segments by partitioning the authorization space into disjoint subspaces. XAnalyzer utilizes APIs provided by Sun XACML 
TABLE 2

XACML Policies Used for Evaluation

\begin{tabular}{|c|c|c|c|}
\hline Policy & Rule (\#) & Policy (\#) & Policy Set (\#) \\
\hline 1 (CodeA) & 4 & 2 & 5 \\
\hline 2 (SamplePolicy) & 6 & 2 & 1 \\
\hline 3 (GradeSheet) & 13 & 1 & 0 \\
\hline 4 (Pluto) & 22 & 1 & 0 \\
\hline 5 (SyntheticPolicy-1) & 147 & 30 & 11 \\
\hline 6 (Continue-a) & 312 & 276 & 111 \\
\hline 7 (Continue-b) & 336 & 305 & 111 \\
\hline 8 (SyntheticPolicy-2) & 456 & 65 & 40 \\
\hline 9 (SyntheticPolicy-3) & 572 & 114 & 75 \\
\hline 10 (SyntheticPolicy-4) & 685 & 188 & 84 \\
\hline
\end{tabular}

implementation [27] to parse the XACML policies and construct Boolean encoding. JavaBDD [15], which is based on BuDDy package [10], is employed by XAnalyzer to support BDD representation and authorization space operations. The effect constraint generation module takes conflicting segments as an input and generates effect constraints for each conflicting segment. Effect constraints are generated based on strategies assigned to each conflicting segment. The strategy mapping module takes conflict correlation groups and effect constraints of conflicting segments as inputs and then maps assigned strategies to standard XACML combining algorithms for examined XACML policy components. The property assignment module automatically assigns corresponding property to each subspace covered by the segments of XACML policy components. The assigned properties are in turn utilized to identify redundancies.

We evaluated the efficiency and effectiveness of XAnalyzer for policy analysis on both real-life and synthetic XACML policies. Our experiments were performed on Intel Core 2 Duo CPU $3.00 \mathrm{GHz}$ with 3.25-GB RAM running on Windows XP SP2. In our evaluation, we utilized five reallife XACML policies, which were collected from different sources. Three of the policies, CodeA, Continue-a, and Continue- $b$ are XACML policies used in [11]; among them, Continue- $a$ and Continue- $b$ are designed for a real-world web application supporting a conference management. GradeSheet is utilized in [7]. The Pluto policy is employed in ARCHON system, ${ }^{3}$ which is a digital library that federates the collections of physics with multiple degrees of metadata richness. In addition, we generated four large synthetic policies SyntheticPolicy-1, SyntheticPolicy-2, SyntheticPolicy-3, and SyntheticPolicy-4 for further evaluating the performance and scalability of our tool. These synthetic policies are multilayered, where each policy component has a randomly selected combining algorithm and each rule has randomly chosen attribute sets from a predefined domain. We also use SamplePolicy, which is the example XACML policy represented in Fig. 1, in our experiments. Table 2 summarizes the basic information of each policy including the number of rules, the number of policies, and the number of policy sets.

We conducted two separate sets of experiments for the evaluation of conflict detection approach and the evaluation of redundancy removal approach, respectively. Also, we performed evaluations at both policy level and policy set level.

3. http://archon.cs.odu.edu/.
TABLE 3 Conflict Detection Algorithm Evaluation

\begin{tabular}{|c|c|c|c|}
\hline \multirow{2}{*}{ Policy } & \multicolumn{3}{|c|}{ Conflict Detection } \\
\cline { 2 - 4 } & Policy Level(\#) & Policy Set Level(\#) & Time (s) \\
\hline 1 (CodeA) & 1 & 1 & 0.082 \\
\hline 2 (SamplePolicy) & 0 & 2 & 0.090 \\
\hline 3 (GradeSheet) & 0 & 4 & 0.098 \\
\hline 4 (Pluto) & 0 & 5 & 0.136 \\
\hline 5 (SyntheticPolicy-1) & 8 & 14 & 0.329 \\
\hline 6 (Continue-a) & 9 & 17 & 0.583 \\
\hline 7 (Continue-b) & 10 & 21 & 0.635 \\
\hline 8 (SyntheticPolicy-2) & 29 & 17 & 0.896 \\
\hline 9 (SyntheticPolicy-3) & 39 & 19 & 0.948 \\
\hline 10 (SyntheticPolicy-4) & 56 & 19 & 1.123 \\
\hline
\end{tabular}

Evaluation of conflict detection. Time required by XAnalyzer for conflict detection highly depends upon the number of segments generated for each XACML policy. The increase of the number of segments is proportional to the number of components contained in an XACML policy. From Table 3, we observed that XAnalyzer performs fast enough to handle larger size XACML policies, even for some complex policies with multiple levels of hierarchies along with hundreds of rules, such as two real-life XACML policies Continue- $a$ and Continue- $b$ and four synthetic XACML policies. The time trends observed from Table 3 are promising and, hence, provide the evidence of efficiency of our conflict detection approach.

Evaluation of redundancy removal. In the second set of experiments, we evaluated our redundancy analysis approach with the same experimental XACML policies in terms of two different cases: redundancy removal considering single-valued requests and redundancy removal considering multivalued requests. Table 4 summarizes that 140 rules were identified as redundant rules in the experimental XACML policies by our redundancy removal approach considering single-valued requests. By comparison, if multivalued requests were taken into account in our redundancy removal algorithm, 21 rules became irremovable. Besides, the evaluation results shown in Table 4 indicate the efficiency of our redundancy analysis algorithm as well.

We also conducted the evaluation of effectiveness by comparing our redundancy analysis approach with a traditional redundancy analysis approach [3], [23], which can only identify redundancy relations between two rules. Fig. 11a depicts the results of our comparison experiments. From Fig. 11a, we observed that XAnalyzer could identify that an average of 5.6 percent of total rules are redundant. However, a traditional redundancy analysis approach could only detect an average 3.1 percent of total rules as redundant rules. Therefore, the enhancement for redundancy elimination was clearly observed by our redundancy analysis approach compared to a traditional redundancy analysis approach in our experiments.

Furthermore, when redundancies in a policy are removed, the performance of policy enforcement is improved generally. For each of XACML policies in our experiments, Fig. 11b depicts the total processing time in Sun XACML PDP [27] for responding 10,000 randomly generated XACML requests. The evaluation results clearly show that the processing times are reduced after eliminating redundancies 
TABLE 4

Redundancy Removal Algorithm Evaluation

\begin{tabular}{|c|c|c|c|c|c|c|}
\hline \multirow{2}{*}{ Policy } & \multicolumn{3}{|c|}{ Redundant Removal Considering Single-valued Requests } & \multicolumn{3}{|c|}{ Redundant Removal Considering Multi-valued Requests } \\
\hline & Policy Level(\#) & Policy Set Level(\#) & Time $(\mathrm{s})$ & Policy Level(\#) & Policy Set Level(\#) & Time $(\mathrm{s})$ \\
\hline $1($ Code $A)$ & 1 & 0 & 0.087 & 1 & 0 & 0.088 \\
\hline 2 (SamplePolicy) & 0 & 2 & 0.095 & 0 & 2 & 0.098 \\
\hline 3 (GradeSheet) & 0 & 2 & 0.113 & 0 & 2 & 0.120 \\
\hline 4 (Pluto) & 0 & 3 & 0.147 & 0 & 3 & 0.151 \\
\hline 5 (SyntheticPolicy-1) & 7 & 4 & 0.158 & 6 & 4 & 0.163 \\
\hline 6 (Continue-a) & 11 & 7 & 0.214 & 8 & 6 & 0.223 \\
\hline 7 (Continue-b) & 12 & 7 & 0.585 & 9 & 7 & 0.597 \\
\hline 8 (SyntheticPolicy-2) & 14 & 8 & 0.623 & 12 & 7 & 0.647 \\
\hline 9 (SyntheticPolicy-3) & 17 & 10 & 0.672 & 15 & 9 & 0.695 \\
\hline 10 (SyntheticPolicy-4) & 23 & 12 & 0.803 & 18 & 10 & 0.852 \\
\hline
\end{tabular}

in XACML policies applying either traditional approach or our approach, and our approach can obtain better performance improvement than the traditional approach.

\section{Related Work}

Many research efforts have been devoted to modeling and verification of XACML policies [2], [8], [11]. In [8], the authors formalized XACML policies using a process algebra known as communicating sequential processes. This work utilizes a model checker to formally verify properties of policies and to compare access control policies with each other. Fisler et al. [11] introduced an approach to represent XACML policies with multiterminal BDDs. They developed a policy analysis tool called Margrave, which can verify XACML policies against the given properties and perform change-impact analysis. Ahn et al. [2] presented a formalization of XACML using answer set programming (ASP), which is a recent form of declarative programming, and leveraged existing ASP reasoners to conduct policy verification. However, lacking an exhaustive elicitation of properties, the completeness of the analysis results of policy verification cannot be guaranteed. In contrast, our approach for policy anomaly analysis can indicate accurate anomaly information without the need of any external properties.

Several works presenting policy analysis tools with the goal of detecting policy anomalies in firewall are closely related to our work. Al-Shaer and Hamed [3] designed a tool called Firewall Policy Advisor that can only detect pairwise anomalies in firewall rules. Yuan et al. [29]

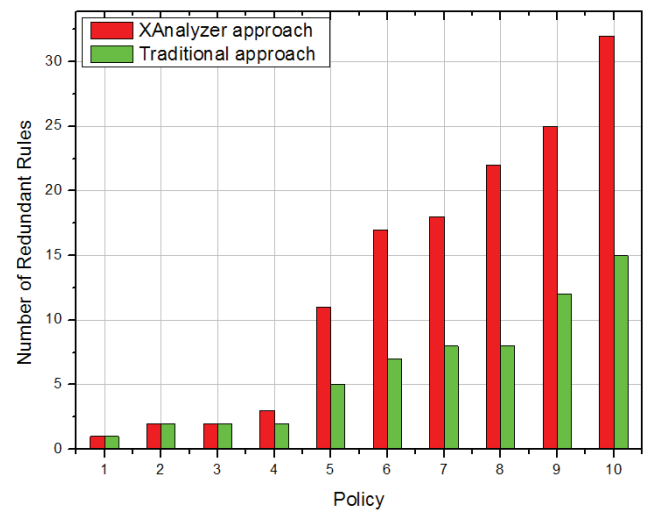

(a) Redundancy elimination rate presented a toolkit, FIREMAN, which can detect anomalies among multiple firewall rules by analyzing the relationships between one rule and the collections of packet spaces derived from all preceding rules. Liu and Gouda [22] introduced a method for complete redundancy detection in firewall rules using a tree representation of firewalls, called firewall decision trees. However, as we discussed previously, due to the significant distinctions between XACML policy and firewall policy, directly applying prior firewall policy anomaly analysis approaches to XACML is not suitable.

Some XACML policy evaluation engines, such as Sun PDP [27] and XEngine [20], [21], have been developed to handle the process of evaluating whether a request satisfies an XACML policy. During the process of policy enforcement, conflicts can be checked if a request matches multiple rules having different effects, and then, conflicts are resolved by applying predefined combining algorithms in the policy. In contrast, our tool XAnalyzer focuses on policy analysis at policy design time. XAnalyzer can identify all conflicts within a policy and help policy designers select appropriate combining algorithms for conflict resolution prior to the policy enforcement. Additionally, XAnalyzer has the capability of discovering and eliminating policy redundancies that cannot be dealt with by policy evaluation engines.

Some works addressed the general conflict resolution mechanisms for access control [11], [14], [16], [17]. Especially, Li et al. [17] proposed a policy combining language PCL, which can be utilized to specify a variety of user-defined

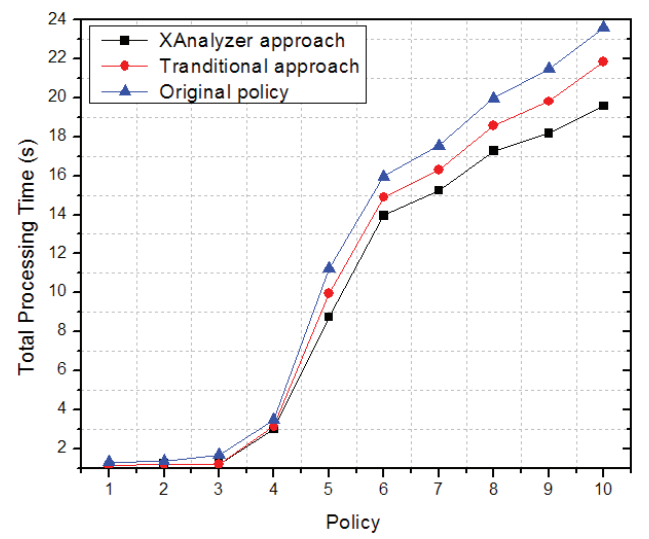

(b) Performance improvement

Fig. 11. Evaluation of a redundancy removal approach. 
combining algorithms for XACML. These conflict resolution mechanisms can be accommodated in our fine-grained conflict resolution framework. In addition, Bauer et al. [6] adopted a data-mining technique to eliminate inconsistencies occurring between access control policies and user's intentions. By comparison, our approach detects and resolves anomalies within access control policies caused by overlapping relations.

Other related work includes XACML policy integration [24], [26] and XACML policy similarity analysis [19]. In particular, Lin et al. [18] designed a comprehensive environment called EXAM for XACML policy analysis and management. EXAM can be used to perform a variety of functions, such as policy property analysis, policy similarity analysis, and policy integration. In contrast, our tool XAnalyzer also deals with policy analysis but focuses on policy anomaly detection and resolution.

\section{Conclusion}

We have proposed an innovative mechanism that facilitates systematic detection and resolution of XACML policy anomalies. A policy-based segmentation mechanism and a grid-based representation technique were introduced to achieve the goals of effective and efficient anomaly analysis. In addition, we have described an implementation of a policy anomaly analysis tool called XAnalyzer. Our experimental results showed that a policy designer could easily discover and resolve anomalies in an XACML policy with the help of XAnalyzer. We believe our systematic mechanism and tool will significantly help policy managers support an assurable web application management service. As our future work, the coverage of our approach needs to be further extended with respect to obligations and userdefined functions in XACML. Moreover, we would explore how our anomaly analysis mechanism can be applied to other existing access control policy languages. In addition, we plan to conduct formal analysis [2], [12] of policy anomalies, particularly dealing with multivalued requests.

\section{ACKNOWLEDGMENTS}

This work was supported in part by the grants from the US National Science Foundation (NSF-IIS-0900970 and NSF-CNS-0831360) and the Department of Energy (DESC0004308).

\section{REFERENCES}

[1] D. Agrawal, J. Giles, K. Lee, and J. Lobo, "Policy Ratification," Proc. Sixth IEEE Int'l Workshop Policies for Distributed Systems and Networks, pp. 223-232, 2005.

[2] G. Ahn, H. Hu, J. Lee, and Y. Meng, "Representing and Reasoning about Web Access Control Policies," Proc. 34th Ann. IEEE Computer Software and Applications Conf., pp. 137-146, 2010.

[3] E. Al-Shaer and H. Hamed, "Discovery of Policy Anomalies in Distributed Firewalls," Proc. IEEE INFOCOM, vol. 4, pp. 26052616, 2004.

[4] J. Alfaro, N. Boulahia-Cuppens, and F. Cuppens, "Complete Analysis of Configuration Rules to Guarantee Reliable Network Security Policies," Int'l J. Information Security, vol. 7, no. 2, pp. 103$122,2008$.

[5] A. Anderson, "Evaluating XACML as a Policy Language," technical report, OASIS, 2003.
[6] L. Bauer, S. Garriss, and M. Reiter, "Detecting and Resolving Policy Misconfigurations in Access-Control Systems," ACM Trans. Information and System Security, vol. 14, no. 1, p. 2, 2011.

[7] A. Birgisson, M. Dhawan, U. Erlingsson, V. Ganapathy, and L. Iftode, "Enforcing Authorization Policies Using Transactional Memory Introspection," Proc. 15th ACM Conf. Computer and Comm. Security, pp. 223-234, 2008

[8] J. Bryans, "Reasoning about XACML Policies Using CSP," Proc. Workshop Secure Web Services, p. 35, 2005

[9] R. Bryant, "Graph-Based Algorithms for Boolean Function Manipulation," IEEE Trans. Computers, vol. C-100, no. 35, pp. 677-691, Aug. 1986.

[10] Buddy, "Buddy Version 2.4, 2010," http://sourceforge.net/ projects/buddy, 2013.

[11] K. Fisler, S. Krishnamurthi, L. Meyerovich, and M. Tschantz, "Verification and Change-Impact Analysis of Access-Control Policies," Proc. 27th Int'l Conf. Software Eng., pp. 196-205, 2005.

[12] H. Hu and G. Ahn, "Enabling Verification and Conformance Testing for Access Control Model," Proc. 13th ACM Symp. Access Control Models and Technologies, pp. 195-204, 2008.

[13] H. Hu, G. Ahn, and K. Kulkarni, "Fame: A Firewall Anomaly Management Environment," Proc. Third ACM Workshop Assurable and Usable Security Configuration, pp. 17-26, 2010.

[14] S. Jajodia, P. Samarati, and V.S. Subrahmanian, "A Logical Language for Expressing Authorizations," IEEE Symp. Security and Privacy, pp. 31-42, May 1997.

[15] JavaBDD, "JavaBDD, 2007," http://javabdd.sourceforge.net, 2013.

[16] J. Jin, G. Ahn, H. Hu, M. Covington, and X. Zhang, "PatientCentric Authorization Framework for Sharing Electronic Health Records," Proc. 14th ACM Symp. Access Control Models and Technologies, pp. 125-134, 2009.

[17] N. Li, Q. Wang, W. Qardaji, E. Bertino, P. Rao, J. Lobo, and D. Lin, "Access Control Policy Combining: Theory Meets Practice," Proc. 14th ACM Symp. Access Control Models and Technologies, pp. 135144,2009

[18] D. Lin, P. Rao, E. Bertino, N. Li, and J. Lobo, "Exam: A Comprehensive Environment for the Analysis of Access Control Policies," Int'l J. Information Security, vol. 9, no. 4, pp. 253-273, 2010.

[19] D. Lin, P. Rao, E. Bertino, and J. Lobo, "An Approach to Evaluate Policy Similarity," Proc. 12th ACM Symp. Access Control Models and Technologies, pp. 1-10, 2007.

[20] A. Liu, F. Chen, J. Hwang, and T. Xie, "XEngine: A Fast and Scalable XACML Policy Evaluation Engine," ACM SIGMETRICS Performance Evaluation Rev., vol. 36, no. 1, pp. 265-276, 2008.

[21] A. Liu, F. Chen, J. Hwang, and T. Xie, "Designing Fast and Scalable XACML Policy Evaluation Engines," IEEE Trans. Computers, vol. 60, no. 12, pp. 1802-1817, Dec. 2011.

[22] A. Liu and M. Gouda, "Complete Redundancy Detection in Firewalls," Proc. 19th Ann. IFIP Conf. Data and Applications Security, 2005.

[23] E. Lupu and M. Sloman, "Conflicts in Policy-Based Distributed Systems Management," IEEE Trans. Software Eng., vol. 25, no. 6, pp. 852-869, Nov./Dec. 1999.

[24] P. Mazzoleni, B. Crispo, S. Sivasubramanian, and E. Bertino, "XACML Policy Integration Algorithms," ACM Trans. Information and System Security, vol. 11, no. 1, article 4, 2008.

[25] T. Moses et al, "Extensible Access Control Markup Language (XACML) Version 2.0," Oasis Standard, 200502, 2005.

[26] P. Rao, D. Lin, E. Bertino, N. Li, and J. Lobo, "An Algebra for FineGrained Integration of XACML Policies," Proc. 14th ACM Symp. Access Control Models and Technologies, pp. 63-72, 2009.

[27] Sun XACML, "Sun XACML Implementation," http://sunxacml. sourceforge.net, 2006.

[28] XACML, "OASIS XACML Committee Website," http://www. oasis-open.org/committees/xacml/, 2011.

[29] L. Yuan, H. Chen, J. Mai, C. Chuah, Z. Su, P. Mohapatra, and C. Davis, "Fireman: A Toolkit for Firewall Modeling and Analysis," Proc. IEEE Symp. Security and Privacy, pp. 199-213, 2006. 


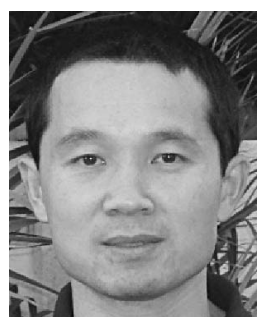

Hongxin $\mathrm{Hu}$ received the $\mathrm{PhD}$ degree in computer science from Arizona State University, Tempe, in 2012. He is an assistant professor in the Department of Computer and Information Sciences at Delaware State University. His current research interests include access control models and mechanisms, security and privacy in social networks, security in cloud and mobile computing, network and system security, and secure software engineering. $\mathrm{He}$ is a member of the IEEE.

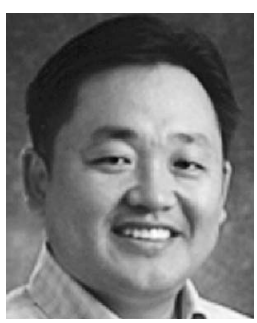

Gail-Joon Ahn received the PhD degree in information technology from George Mason University, Fairfax, Virginia, in 2000. He is an associate professor in the School of Computing, Informatics, and Decision Systems Engineering, Ira A. Fulton Schools of Engineering and the director of Security Engineering for Future Computing Laboratory, Arizona State University. His research has been supported by the US National Science Foundation, the National Security Agency, the US Department of Defense, the US Department of Energy, Bank of America, Hewlett Packard, Microsoft, and the Robert Wood Johnson Foundation. He received the US Department of Energy CAREER Award and the Educator of the Year Award from the Federal Information Systems Security Educators Association. $\mathrm{He}$ is a senior member of the IEEE.

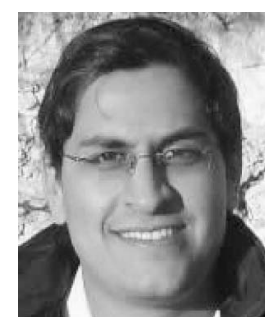

Ketan Kulkarni received the master's degree in computer science from Arizona State University. $\mathrm{He}$ was also a member of the Security Engineering for Future Computing Laboratory, Arizona State University. $\mathrm{He}$ is currently a software engineer at NVIDIA.

For more information on this or any other computing topic, please visit our Digital Library at www.computer.org/publications/dlib. 